\title{
Autophagy in aging, disease and death: the true identity of a cell death impostor
}

\author{
B Levine ${ }^{\star, 1,2,3}$ and G Kroemer ${ }^{\star, 4,5,6}$ \\ Cell Death and Differentiation (2009) 16, 1-2; doi:10.1038/cdd.2008.139
}

Although strictly speaking, the term autophagy merely means 'self-eating', many presume that this cellular self-eating is inevitably a form of cellular self-destruction. Indeed, within the cell death research field, autophagy has also long been defined as a form of non-apoptotic, or type II, programmed cell death. However, as revealed in this issue of Cell Death and Differentiation, which contains nine review papers on the topic of Autophagy in Aging, Disease and Death ${ }^{1-9}$ and one article outlining the recommendations of the Nomenclature Committee on Cell Death $2009,^{10}$ a consensus is emerging that autophagy is largely a cell death impostor which, in reality, functions primarily to promote cellular and organismal health.

Five of the papers in this series describe unequivocal beneficial functions of autophagy in the life of a cell or eukaryotic organism. Sarkar et al. ${ }^{1}$ discuss the beneficial effects of mTOR-dependent and mTOR-independent induction of autophagy in enhanced neuronal clearance and reduced neurotoxicity of mutant huntingtin fragment and other polyglutamine expansion proteins in cultured cells and animal models. Perlmutter ${ }^{2}$ discusses the role of autophagy in the disposal of mutant $\alpha$-1-antitrypsin, a toxic aggregate-prone protein that accumulates in the endoplasmic reticulum of hepatocytes and causes liver inflammation and carcinogenesis. Orvedahl and Levine ${ }^{3}$ discuss the immune signaling mechanisms that activate autophagy, the different effector mechanisms of autophagy in immunity, and the potential for exploiting the autophagy pathway in the treatment of infectious diseases. Lunemann and Munz ${ }^{4}$ provide a more focused review on one important aspect of autophagy in immunity, namely, its role in delivering cytoplasmic material to MHC Class II antigen-loading compartments for regulation of adaptive immune responses. Finally, Vellai ${ }^{5}$ discusses the compelling genetic evidence in Caenorhabditis elegans and Drosophila models that autophagy functions as an antiaging mechanism, presumably by degrading aberrant cytosolic macromolecules and organelles.

Thus, the delivery function of autophagy - either to the lysosome for degradation or to other cellular compartments for immune activation - is important in protection against aging, neurodegenerative diseases, $\alpha$-1-antitrypsin deficiency, and infectious diseases and indeed, clinical trials with autophagy-inducing agents are now being planned for the prevention of aging, and treatment of certain neurodegenerative and infectious diseases. In the case of lysosomal delivery, 'self-eating' (as well as xenophagy, the digestion of microbes) certainly does occur. However, it is not part of a program of cellular self-destruction that leads to cell death. Rather, it is a mechanism by which the cell rids itself of potentially harmful constituents, and thereby helps to maintain its normal functioning. Thus, after years of being (mis)interpreted as a cell death process, the study summarized in the review papers in this issue shed important light on the true identity of autophagy - which is, in part, an adaptive cellular housekeeping mechanism.

Two other papers in this review series describe a more complex interplay between autophagy and disease, in particular, cancer and heart disease, where autophagy may have context-dependent beneficial or detrimental roles. ${ }^{6,7}$ Maiuri et al. ${ }^{6}$ review the emerging paradigm that oncogenes inhibit autophagy, whereas tumor-suppressor genes induce autophagy, as well as a notable exception to this paradigm, which is the inhibition of autophagy by the cytoplasmic form of the tumor-suppressor protein, p53. The authors propose that chronic suppression of autophagy promotes oncogenesis, perhaps through genomic instability, defective cell growth regulation, and/or defective regulation of endogenous genotoxic stress. However, they also note that enhanced autophagy may constitute a mechanism utilized by tumor cells to survive hypoxic, metabolic, detachment-induced, or chemotherapeutic stress. Thus, ironically, the malevolent function of autophagy in cancer, if it has one, is not a role in cell death, but rather, a role in tumor cell survival. Given the duality of autophagy's function in tumor suppression and tumor cell survival, as the authors' state, the 'relationship between cancer and autophagy cannot be reduced to a simple formulation' and further studies are needed to determine if, when, and how clinical oncologists should turn autophagy on or off.

\footnotetext{
${ }^{1}$ Howard Hughes Medical Institute, University of Texas Southwestern Medical Center, 5323 Harry Hines Boulevard, Dallas, TX 75390, USA; ${ }^{2}$ Department of Internal Medicine, University of Texas Southwestern Medical Center, 5323 Harry Hines Boulevard, Dallas, TX 75390, USA; ${ }^{3}$ Department of Microbiology, University of Texas Southwestern Medical Center, 5323 Harry Hines Boulevard, Dallas, TX 75390, USA; ${ }^{4}$ Institut Gustave Roussy, F-94805 Villejuif, France; ${ }^{5}$ Université Paris Sud, Paris 11 , F-94805 Villejuif, France and ${ }^{6}$ INSERM, U848, F-94805 Villejuif, France

${ }^{*}$ Corresponding authors: B Levine, Division of Infectious Diseases, University of Texas Southwestern Medical Center, 5323 Harry Hines Boulevard, Dallas, TX 753909113, USA. Tel: 214648 0493; Fax: 214648 0284; E-mail: beth.levine @utsouthwestern.edu or G Kroemer, INSERM, U848, Institut Gustave Roussy, Pavillon de Recherche 1, 39 rue Camille-Desmoulins, Villejuif F-94805, France. Tel: +33 142116046 ; Fax: + 331421160 47; E-mail: kroemer@igr.fr
} 
In the heart, it is still unclear whether autophagy is a protective or detrimental response in responses to stresses such as ischemia/reperfusion and in cardiovascular diseases such as cardiac hypertrophy and heart failure. Nishida et al. ${ }^{7}$ provide a comprehensive review of this controversy, highlighting the evidence from cardiac-specific atg5-deficient mice that autophagy protects cardiomyocytes from pressure overlaod or from isoproterenol stimulation (which contrasts with evidence from beclin 1-deficient mice) and the conflicting evidence that autophagy may mediate either cell survival or cell death during ischemia/reperfusion. Future studies in mouse models with cardiomyocyte-deficient autophagy may be useful in delineating the functions of autophagy in heart disease. However, given the limitations of mouse models of cardiac disease, a greater challenge will be to figure out if, when, and how cardiologists should regulate autophagy in patients with heart disease.

With the exception of the mention of a possible role of excessive autophagy in the death of cardiomyocytes during ischemia/reperfusion injury, the papers in this issue that deal with autophagy in aging or disease underscore an identity of autophagy that is quite distinct from a cell death execution pathway. The picture that emerges is that the 'core identity' of autophagy is one of a cellular pathway that is cytoprotective. The pathway protects cells against the accumulation of damaged organelles, protein aggregates, and microbes; protects cells against oncogenesis; protects cells against cancer therapies, protects cardiac cells against hemodynamic stress; and protects infected cells by activating immune defenses. Thus, the frequent presence of autophagy in dying cells may represent a failed attempt of cytoprotection, rather than a mechanistic contribution to cell death; hence, we propose that autophagy is largely a 'cell death impostor.'

Yet, the controversy remains as to whether in some circumstances autophagy is not merely a cell death impostor, but a bona fide mechanism of cell death. The Nomenclature Committee on Cell Death 2009 defines 'autophagic cell death' as a set of morphological features that 'define cell death occurring with autophagy,' noting that this term 'may misleadingly suggest a form of death occurring through autophagy, as this process often promotes cell survival. ${ }^{10}$ However, two papers in this issue review several examples of cell death by (not just with) autophagy; Scarlatti et al. ${ }^{8}$ discuss the evidence in mammalian cells and Kourtis and Tavernakis discuss the evidence in model organisms. ${ }^{8,9}$

In cultured mammalian cells, cell death by canonical autophagy (defined as death that is reduced by genetic inactivation of autophagy genes including beclin 1) has been reported primarily (but not exclusively) in cells that are deficient in apoptosis, either by the virtue of bax/bak deletion or caspase inhibition. Autophagy has also been described to be genetically upstream of apoptosis in the setting of HIV envelope protein triggered T lymphocyte death. Further, some death-inducing stimuli, such as the Parkinson neurotoxin, 1-methyl-4-phenylpyridiunium, and resveratrol reportedly induce cell death by non-canonical autophagy, which is independent of beclin 1 (a gene involved in autophagosome initiation), but requires other autophagy genes involved in autophagosome expansion and completion. In vivo, there are two examples to date in model organisms where knockdown/ knockout of autophagy genes retards cell death, in the involuting Drosophila melanogaster salivary gland and in nematodes with hyperactive ion channels that undergo necrotic neuronal cell death. Direct induction of autophagy by overexpression of the Atg1 kinase has also been shown to be sufficient to kill fat and salivary gland cells in Drosophila.

It is not yet clear how to reconcile the few examples of 'cell death by autophagy' with the larger number of studies in which autophagy suppression by genetic knockout/knockdown of essential autophagy genes increases cell death (reviewed in Scarlatti et al., ${ }^{8}$ Kourtis and Tavernakis ${ }^{9}$ and Maiuri et al. ${ }^{11}$ ), indicating a prosurvival function of autophagy. Further, as noted by Scarlatti et al., ${ }^{8}$ the evidence for cell death by autophagy remains to be demonstrated in mammals; in fact, embryonic mice lacking autophagy genes, including ambra1, beclin 1, and atg5, have been shown to have increased, not decreased, numbers of apoptotic cells (reviewed in Cecconi and Levine ${ }^{12}$ ). Thus, although it is certainly possible that autophagy is not always a cell death impostor, the majority of studies discussed in this issue of Cell Death and Differentiation (and recently reviewed elsewhere) suggest that the primary identity of autophagy lies elsewhere, namely, in cytoprotection. It will be important to further unravel the mysteries of the complex interplay between autophagy and cell death, but arguably, it may be even more important to further unravel the relationships between autophagy, aging, disease, and cell survival. The review papers in this series provide an excellent point of departure for meeting both of these scientific challenges.

1. Sarkar S, Andres Floto R, Rubinsztein D. Rapamycin and mTOR-independent autophagy inducers ameliorate toxicity of polyglutamine huntingtin and related proteinopathies. Cell Death Diff 2008; 16: 46-56.

2. Perlmutter D. Autophagic disposal of the aggregation-prone protein that causes liver inflammation and carcinogenesis in $\alpha$-1-antitrypsin deficiency. Cell Death Diff 2008; 16 : $39-45$.

3. Orvedahl A, Levine B. Eating the enemy within: autophagy in infectious diseases. Cell Death Diff 2008; 16: 57-69.

4. Lunemann J, Munz C. Autophagy in $\mathrm{CD}^{+} \mathrm{T}$-cell immunity and tolerance. Cell Death Diff 2008; 16: 79-86.

5. Vellai T. Autophagy genes and ageing. Cell Death Diff 2008; 16: 94-102.

6. Maiuri M, Tazdemir E, Criollo A, Morselli E, Vicencio J, Carnuccio R et al. Control of autophagy by oncogenes and tumor suppressor genes. Cell Death Diff 2008; 16: 87-93.

7. Nishida K, Kyoi S, Yamaguchi O, Sadoshima J, Otsu K. The role of autophagy in the heart Cell Death Diff 2008; 16: 31-38.

8. Scarlatti F, Granata R, Meijer A, Codogno P. Does autophagy have a license to kill mammalian cells? Cell Death Diff 2008; 16: 12-20.

9. Kourtis N, Tavernakis N. Autophagy and cell death in model organisms. Cell Death Diff 2008; 16: 21-30.

10. Kroemer G, Galluzi L, Vandenabeele P, Abrams J, Alnemri E, Baehrecke E et al. Classification of cell death: recommendations of the Nomenclature Committee on Cell Death 2009. Cell Death Diff 2008; 16: 3-11.

11. Maiuri M, Zalckvar E, Kimchi A, Kroemer G. Self-eating and self-killing: crosstalk between autophagy and apoptosis. Nat Rev Mol Cell Biol 2007; 8: 741-752.

12. Cecconi F, Levine B. The role of autophagy in mammalian development: cell makeove rather than cell death. Dev Cell 2008; doi:10.1016/j.devcel.2008.08.012. 